María del Pilar Sánchez-Muñoz

José Gabriel Cruz-Cerón

Recibido: 19 de noviembre de 2018 Concepto de evaluación: 20 de febrero de 2019

Aprobado: 12 de diciembre de 2019

Artículo de investigación (c) 2019 Universidad Católica de Colombia. Facultad de Ciencias Económicas y Administrativas. Todos los derechos reservados

* Doctora en Desarrollo Sostenible. Docente de la Facultad de Economía de la Universidad del Rosario (Bogotá, Colombia). Investigadora del Grupo Economía y Desarrollo Humano de la Universidad de La Salle. Correo electrónico: madpilarsanchez@gmail.com

(D) http://orcid.org/0000-0002-9325-9091

** Doctor en Ciencias Económicas. Profesor titular e investigador de la Universidad de Caldas (Manizales).

Correo electrónico: gabocruzc@gmail.com

(D) http://orcid.org/0000-0003-1550-6517

*** Economista de la Universidad de La Salle. Analista en Supply Chain en Centurylink Colombia (Bogotá, Colombia).

Correo electrónico: carolina.maldonado1990@gmail.com

(D) http://orcid.org/0000-0003-0107-6659
Finanz. polit. econ., ISSN: 2248-6046, Vol. 11, N. ${ }^{\circ}$ 2, julio-diciembre, 2019, pp. 321-336

http://doi.org/10.14718/revfinanzpolitecon.2019.11.2.6

\section{Gestión de residuos sólidos urbanos en América Latina: un análisis desde la perspectiva de la generación}

\section{RESUMEN}

La adecuada gestión de los residuos sólidos es agenda de trabajo para el desarrollo sostenible. América Latina ha adoptado políticas y promulgado normatividad que han llevado a la prohibición de botaderos a cielo abierto, la incorporación de los recicladores al proceso de gestión y el intento de reducir la cantidad de residuos generados. En este artículo se analiza la correlación entre la generación de residuos sólidos urbanos y algunas variables en nueve ciudades de la región, entre el 2007 y el 2014, utilizando estadísticas oficiales de cada país. Se encuentra que el ingreso por habitante y la tasa de cobertura escolar se correlacionan significativamente. Se concluye la importancia de generar conciencia en los ciudadanos respecto a su papel en la reducción de residuos sólidos generados.

Palabras clave: desarrollo sostenible, educación ambiental, residuos sólidos urbanos, territorio.

\section{JEL Classification: Q01, Q53, Q58}

Cómo citar este artículo / To reference this article / Para citar este artigo:

Sánchez-Muñoz, M., P., Cruz-Cerón, J., G. \& Maldonado-Espinel, P., C. (2019). Gestión de residuos sólidos urbanos en América Latina: un análisis desde la generación. Revista Finanzas y Politica Económica, 11(2), 321-336. doi: http://dx.doi.org/10.14718/ revfinanzpolitecon.2019.11.2.6

\section{Urban solid waste management in Latin America:} An analysis from the perspective of waste generation

\section{ABSTRACT}

Solid waste management is a working agenda for sustainable development. Latin America has adopted policies and promulgated regulations that have led to the prohibition of open-air dumps, an inclusion of recyclers into the management process, and incentives to reduce the generation of urban solid waste. This article analyzes the correlation between the generation of urban solid waste and some other factors in nine cities of the region, between 2007 and 2014, using official statistics from each country. Results show that there is a significant correlation between per capita income and school coverage rate. Although there have been some improvements, there also remain challenges 
in order to raise awareness among citizens about their role in reducing the generation of solid waste.

Keywords: Environmental education, sustainable development, territory, urban solid waste.

\section{Gestão de resíduos sólidos urbanos na América Latina: uma análise a partir da geração}

\section{RESUMO}

A adequada gestão dos resíduos sólidos é agenda de trabalho para o desenvolvimento sustentável. A América Latina tem adotado políticas e promulgado regulamentações que levam à proibição de aterros a céu aberto, à incorporação dos recicladores ao processo de gestão e à tentativa de reduzir a quantidade de resíduos gerados. Neste artigo, a correlação entre a geração de resíduos sólidos urbanos e algumas variáveis é analisada em nove cidades da região, entre 2007 e 2014, com a utilização de estatísticas oficiais de cada país. Constata-se que o ingresso por habitante e a taxa de cobertura escolar se correlacionam de forma significativa. Conclui-se que é importante conscientizar os cidadãos quanto a seu papel na redução de resíduos sólidos gerados.

Palavras-chave: desenvolvimento sustentável, educação ambiental, resíduos sólidos urbanos, território. 


\section{INTRODUCCIÓN}

El medioambiente se ha convertido en discusión obligada en los ámbitos nacional, local, público y privado. La preocupación por un mundo posible para las generaciones futuras, y el aporte de cada persona, ciudad, país y región para conseguirlo, se han traducido en acciones y enfoques tendientes al logro de metas comunes en temas como agua, aire o suelo, hoy sintetizados en los Objetivos de Desarrollo Sostenible (ODS).

Uno de ellos es el de lograr una adecuada gestión de los residuos sólidos, proceso que cobra importancia cuando la dinámica poblacional de la humanidad evidencia una cada vez mayor aglomeración en grandes urbes, lo cual exige un mejor manejo de los desechos para no generar mayor impacto en la atmósfera (por ejemplo, por el metano producido) o en el suelo y el agua (como sucede con la contaminación por lixiviados, al disponer los residuos en rellenos sanitarios, caso generalizado en América Latina).

En el hemisferio se han dado avances para reducir estos efectos prohibiendo los botaderos a cielo abierto, incorporando a los recicladores al proceso y tratando de reducir la cantidad de residuos generados. En este artículo se analiza la generación de residuos sólidos urbanos por habitante y su relación con variables que se consideran relevantes, como el ingreso per cápita y las tasas de cobertura en educación.

Cabe resaltar la importancia de hacer el análisis de las variables seleccionadas en el contexto propio de la región, donde se han establecido políticas de manejo de residuos sólidos tanto en el ámbito nacional como local, traducidas en un conjunto de normativas específicas y en algunas acciones tendientes a minimizar la generación, en un contexto donde la basura es residuo que puede ser reincorporado al ciclo productivo de bienes $\mathrm{y}$ servicios.

La información requerida se obtiene de las fuentes oficiales de la Ciudad Autónoma de Buenos Aires, Belo Horizonte, Curitiba, Porto Alegre, Río de Janeiro, Región Metropolitana de Santiago, Bogotá
D.C., Medellín y Lima Metropolitana. Se toma el periodo 2007-2014, dado que no hubo posibilidad de acceder a datos de años anteriores.

Para el desarrollo de la investigación se utiliza estadística descriptiva y análisis correlacional, y se aplican pruebas de normalidad y coeficiente de Pearson, a partir de lo cual se identifica que no es posible generalizar las relaciones existentes entre las ciudades estudiadas. El artículo compara durante ocho años el comportamiento de la generación de residuos sólidos en las ciudades seleccionadas y analiza si las variables de una u otra manera están relacionadas con este. Igualmente, busca aprender de los casos en los cuales la normativa no se quedó solo en el deber ser, sino que a través de acciones particulares logró cambios significativos.

El documento está dividido en seis partes, de las que esta introducción es la primera. En la segunda se hace una reflexión teórica con énfasis en la necesidad de construir la política pública desde lo local. En la tercera se presenta la metodología. En la cuarta se esbozan los elementos institucionales que enmarcan la preocupación de la región por regular la gestión de los residuos sólidos urbanos. En la quinta se despliega el análisis descriptivo y correlacional de las variables. Finalmente se presentan las conclusiones pertinentes.

\section{LOS RESIDUOS SÓLIDOS URBANOS EN EL MARCO DEL DESARROLLO LOCAL Y SOSTENIBLE}

El concepto de desarrollo ha evolucionado a lo largo de la historia, debido a la necesidad de dar solución a múltiples problemáticas de la sociedad. Dado que el mundo se estudia desde sus particularidades, el término desarrollo local se ha vuelto relevante a la hora de analizar y ofrecer alternativas de mejora. En concreto, Tello (2006) define el concepto de esta manera:

[Es] el proceso de la dinámica económica, social y política de un área geográfica específica resultante del comportamiento, 
acciones e interacciones de los agentes (económicos, políticos y sociales), [...] que tiene la finalidad de incrementar sostenida y sosteniblemente el nivel y la calidad de vida de los habitantes de dicha área geográfica usando plena y eficientemente sus recursos humanos y no humanos. (p. 11)

Igualmente, se ha acuñado el concepto de desarrollo sostenible a partir de la reflexión sobre el efecto de las actividades antrópicas en el entorno. Como lo señala el Informe Brudtland, "el desarrollo sostenible es aquel que satisface las necesidades presentes sin comprometer las futuras generaciones" (Comisión Mundial sobre Medio Ambiente y el Desarrollo, 1987). Cuando se habla de lo local, se piensa en un conjunto limitado de elementos (espacio, capacidad, personas), y esto, desde una visión biocéntrica, hace más consciente al ser humano de las decisiones que toma y lo vuelven más consecuente con las acciones que realiza para causar menos efectos negativos en el ambiente, una mayor calidad de vida y una reducción de las inequidades entre las comunidades.

Ahora bien, el manejo adecuado de residuos sólidos urbanos ${ }^{1}$ es un factor determinante para mejorar las condiciones medioambientales de las ciudades, con repercusiones favorables para todos los agentes de la sociedad. Como lo plantean Usaquén y Sánchez (2014), esta práctica ayuda a generar empleo y a reducir la pobreza. Y desde el enfoque de Sen (2001) consiste en satisfacer las necesidades básicas y mejorar las oportunidades de acceso al mercado.

En los últimos años algunos países de América Latina han involucrado la gestión de los residuos sólidos urbanos en su agenda política. Para ello, han planteado un conjunto de directrices y normativas que buscan cambiar la actitud de todos los

1 "Residuos generados en los hogares y sus asimilables, como los residuos generados en vías públicas, el comercio, oficinas, edificios e instituciones tales como escuelas, entre otros. Estos residuos son considerados residuos no peligrosos" (Gobierno de Chile, Comisión Nacional de Medio Ambiente [CONAMA], 2010, p. 11). miembros de la comunidad, reducir la cantidad generada de estos residuos y mitigar la presión sobre el medioambiente. En palabras de Dueñas (2011), "debemos cambiar radicalmente nuestra actitud hacia los aspectos socioambientales. Esta actitud debe ser activa, participativa, coherente y responsable. Los ciudadanos debemos ser protagonistas de esta nueva cultura" (p. 17).

Además, se hace necesario enfatizar en la educación ambiental para todos los ciudadanos; una educación que ponga en diálogo las necesidades no satisfechas de los individuos, la preservación de los recursos naturales y el logro de un verdadero desarrollo.

\section{METODOLOGÍA}

Para el desarrollo de la investigación se utilizó estadística descriptiva y análisis correlacional, y se aplicó la prueba de normalidad Kolmogorov-Smirnov, la cual determina que el coeficiente de Pearson es la mejor medida del grado de asociación lineal existente entre las variables. Adicionalmente, se obtuvo información de organismos oficiales de cada país en torno a la generación de residuos sólidos urbanos por habitante y sobre variables como el ingreso per cápita y las tasas de cobertura en educación en algunas ciudades de la región (tabla 1). Las ciudades seleccionadas fueron: Ciudad Autónoma de Buenos Aires, Belo Horizonte, Curitiba, Porto Alegre, Río de Janeiro, Región Metropolitana de Santiago, Bogotá D.C., Medellín y Lima Metropolitana. Se toma el periodo 2007-2014, dado que no hubo posibilidad de acceder a datos de años anteriores en las fuentes oficiales consultadas.

\section{INSTITUCIONALIDAD DE LA GENERACIÓN DE RESIDUOS SÓLIDOS URBANOS EN CIUDADES DE AMÉRICA LATINA}

En este apartado se esbozan los avances que han tenido los países seleccionados en materia de instituciones formales para incentivar las buenas 
Tabla 1.

Fuentes secundarias de ciudades latinoamericanas

\begin{tabular}{|c|c|}
\hline Ciudad & Procedencia de la información \\
\hline $\begin{array}{l}\text { Ciudad Autónoma de Buenos } \\
\text { Aires }\end{array}$ & $\begin{array}{c}\text { Área Metropolitana CEMSE ecología urbana; Dirección General de Estadística y Censos (Ministerio de } \\
\text { Hacienda GCBA); INDEC, Serie Análisis Demográfico n.os } 31 \text { y 36; y Dirección General de Estadística y } \\
\text { Censos (Ministerio de Hacienda GCBA), EAH 2002/2014. }\end{array}$ \\
\hline $\begin{array}{l}\text { Belo Horizonte, Curitiba, Porto } \\
\text { Alegre, Río de Janeiro }\end{array}$ & $\begin{array}{l}\text { Secretaría Nacional de Saneamiento Ambiental [SNSA], Ministério das Ciudades; IBGE, Diretoria de } \\
\text { Pesquisas, Coordenação de População e Indicadores Socias; y Censo Escolar (2000-2014). }\end{array}$ \\
\hline $\begin{array}{c}\text { Región Metropolitana de } \\
\text { Santiago }\end{array}$ & $\begin{array}{c}\text { Ministerio del Medio Ambiente (MMA); Banco Central de Chile, Base de Datos Estadísticos; INE, } \\
\text { Demográficas y vitales; y (2015) Ministerio de Desarrollo Social, Informe de desarrollo Social, } \\
\text { Ministerio de Desarrollo Social, Encuesta CASEN años respectivos. }\end{array}$ \\
\hline Bogotá D.C., Medellín & $\begin{array}{l}\text { Sistema Único de Información (SUI); Unidad Administrativa Especial de Servicios Públicos; } \\
\text { Observatorio Ambiental de Bogotá; DANE, Cuentas Departamentales; Departamento Administrativo } \\
\text { de Planeación; Alcaldía de Medellín; DANE, Estimaciones de Población 1985-2005 y Proyecciones de } \\
\text { Población 2005-2020; Secretaría de Educación Distrital, Boletín General; y Secretaria de Educación de } \\
\text { Medellín. }\end{array}$ \\
\hline Lima Metropolitana & $\begin{array}{c}\text { Municipalidad Metropolitana de Lima, Gerencia de Servicios a la Ciudad, Sub- Gerencia de Medio } \\
\text { Ambiente, División de Gestión de Residuos Sólidos; Municipalidad Provincial del Callao; Instituto } \\
\text { Nacional de Estadística e Informática, Encuesta Nacional de Hogares; e INEI, Encuesta Nacional de } \\
\text { Hogares ENAHO 2004-2012. }\end{array}$ \\
\hline
\end{tabular}

Fuente: elaboración propia.

prácticas alrededor de los residuos sólidos urbanos. A comienzos del siglo XXI empieza a crearse la normatividad sobre el adecuado manejo de este tipo de residuos en las ciudades seleccionadas. No obstante, infortunadamente en la mayoría de ellas tales disposiciones y políticas no se han materializado en transformaciones de sus propias realidades, ni en cambios de comportamientos de los ciudadanos para hacer un consumo responsable y así reducir la generación de residuos. Ciertamente, se observa la vinculación del tema dentro de la institucionalidad, pero en términos prácticos los planes de acción han estado más enfocados a organizar los aspectos logísticos y en menor medida a lograr la minimización de la cantidad de residuos generados.

En coherencia con lo planteado por el Banco Interamericano de Desarrollo (BID), se encuentra que la región ha avanzado en la institucionalidad del sector a través de programas de separación en fuente y reciclaje. Sin embargo, las leyes "son generalmente redactadas a nivel marco, su aporte específico es leve y su cumplimiento bajo, ya que rara vez se logran reglamentar. Incluso reglamentadas, la falta de aplicación de los mecanismos de control y sanción previstos atenta contra su efectividad" (BID et al., 2011, p. 73).
Conviene resaltar que en toda Latinoamérica las directrices nacionales no han deslegitimado que desde el ámbito de cada municipalidad se apliquen acciones que permitan cumplir los objetivos en cuanto al manejo de los residuos sólidos. De este modo, refuerzan la búsqueda de un verdadero desarrollo local, es decir, "un proceso de cambio estructural localizado que se asocia a un permanente proceso de progreso de la propia región, de la comunidad o sociedad que habita en ella y de cada individuo miembro de tal comunidad y habitante de tal territorio" (Boisier, 1999, p. 7).

En la tabla 2 se resumen las principales normas tanto a nivel nacional como de cada una de las ciudades analizadas, incluyendo algunos de los programas implementados y leyes que en materia de educación ambiental existen para promover la reducción. Algunas ciudades como Monterrey y Medellín han establecido programas específicos para el tratamiento de los residuos sólidos, como "Unidos por la Limpieza - Programa Nuevo León Recicla" y "Ecohuertas Urbanas", respectivamente. En el caso de Monterrey, "cuenta con algunos servicios instalados para reciclaje, con puntos centralizados de recolección y la capacidad de reciclar desechos orgánicos, vidrios, plástico y papel. 
La ciudad cuenta también con una política integral que tiene por objeto reducir, reutilizar y reciclar los desechos" (Economist Intelligence Unit, 2010, p. 70). Sin embargo, no hay evidencia de la existencia de programas que incluyan el manejo de los residuos sólidos como un sistema.

Aunque también se orientan a la educación ambiental, en Lima "existen muy pocos programas para difundir la cultura ambiental, programas de educación ambiental orientados a desarrollar conciencia de corresponsabilidad ambiental y prácticas de reducción, reúso y reciclaje de residuos sólidos en la población" (Municipalidad Metropolitana de Lima, 2014, p. 30). Si bien existen programas como "Escuelas Verdes", sigue habiendo la necesidad de control y monitoreo, a lo que se suma la falta de compromiso de los generadores frente a la participación activa en estos procesos.

Pese a lo anterior, se rescata que Lima limita en su estructura normativa, a través del Decreto 147 de 2001, la generación máxima de residuos y responsabiliza al generador de la disposición final de la cantidad que exceda. En este mismo sentido, la Ciudad Autónoma de Buenos Aires establece topes máximos de generación de residuos sólidos en coherencia con la Ley 1854 de 2005, en la cual se adopta el concepto de "Basura Cero". Aunque los resultados esperados por la ley no se visualizaron en el tiempo establecido, la cantidad de residuos sólidos urbanos generados en promedio por cada habitante se redujo en un $40 \%$ entre el 2012 y el 2014, al pasar de 1,31 a 0,79 ton/año. En palabras de Greenpeace (2014), "más allá del enorme retraso en la gestión integral de la basura y de falencias que hoy en día persisten, la reducción [...] da muestras suficientes de la factibilidad de la Ley".

Si bien cada ciudad reporta avances en su normativa y son tímidas las acciones concretas, Curitiba es ejemplo de avance conjunto en estos dos aspectos, ya que ha formulado políticas y leyes direccionadas a la mejora continua de la gestión, incluyendo programas como "Basura que no es Basura” y "Compra de Basura”, instituidos hace más de veinte años y que han reforzado la conciencia ambiental de los ciudadanos. De acuerdo con Mullahy (1993), "ambas iniciativas se basan en un sistema de incentivos, en la participación de la comunidad local y en la búsqueda de la simplicidad: la facilidad de uso de estos sistemas ha permitido una rápida aceptación por parte de la población" (p.63).

Debe resaltarse que las ciudades de Brasil fueron pioneras en la consolidación de instituciones formales propias de cada municipalidad, que luego se articularon a través de la Política Nacional de Residuos Sólidos con la Ley 12.305 de 2010. Este caso muestra la importancia de construir la dinámica económica, social y política desde lo local, con miras a mejorar la calidad de vida de los ciudadanos, pues así es posible estar más cerca de sus problemáticas y las posibles soluciones.

Conscientes de que parte del manejo adecuado de los residuos sólidos consiste en la separación en la fuente y la disposición adecuada, ciudades como Belo Horizonte, Región Metropolitana de Santiago y Bogotá han aportado al reconocimiento de la labor que realizan los recolectores de material potencialmente reutilizable, dignificando así su trabajo y adoptando medidas para su inclusión social.

\section{AMÉRICA LATINA EN CIFRAS}

Por disponibilidad de la información estadística, en esta sección se realiza un análisis descriptivo, entre el 2007 y el 2014, del PIB per cápita a PPA, las tasas de cobertura de educación básica y media y el coeficiente de Gini. Estas variables están relacionadas con la cantidad de toneladas de residuos sólidos urbanos generadas cada año por habitante en la ciudades de referencia. Cabe aclarar que la generación de residuos sólidos urbanos se relaciona además con variables como la actividad económica, los patrones de consumo, la relación urbano-rural y la densidad poblacional; sin embargo, las ciudades no cuentan con información estadística que permita un análisis cuantitativo a este respecto.

Usaquén y Sánchez (2014) elaboraron un modelo de datos panel para algunos municipios de Cundinamarca (Colombia) y encontraron que 


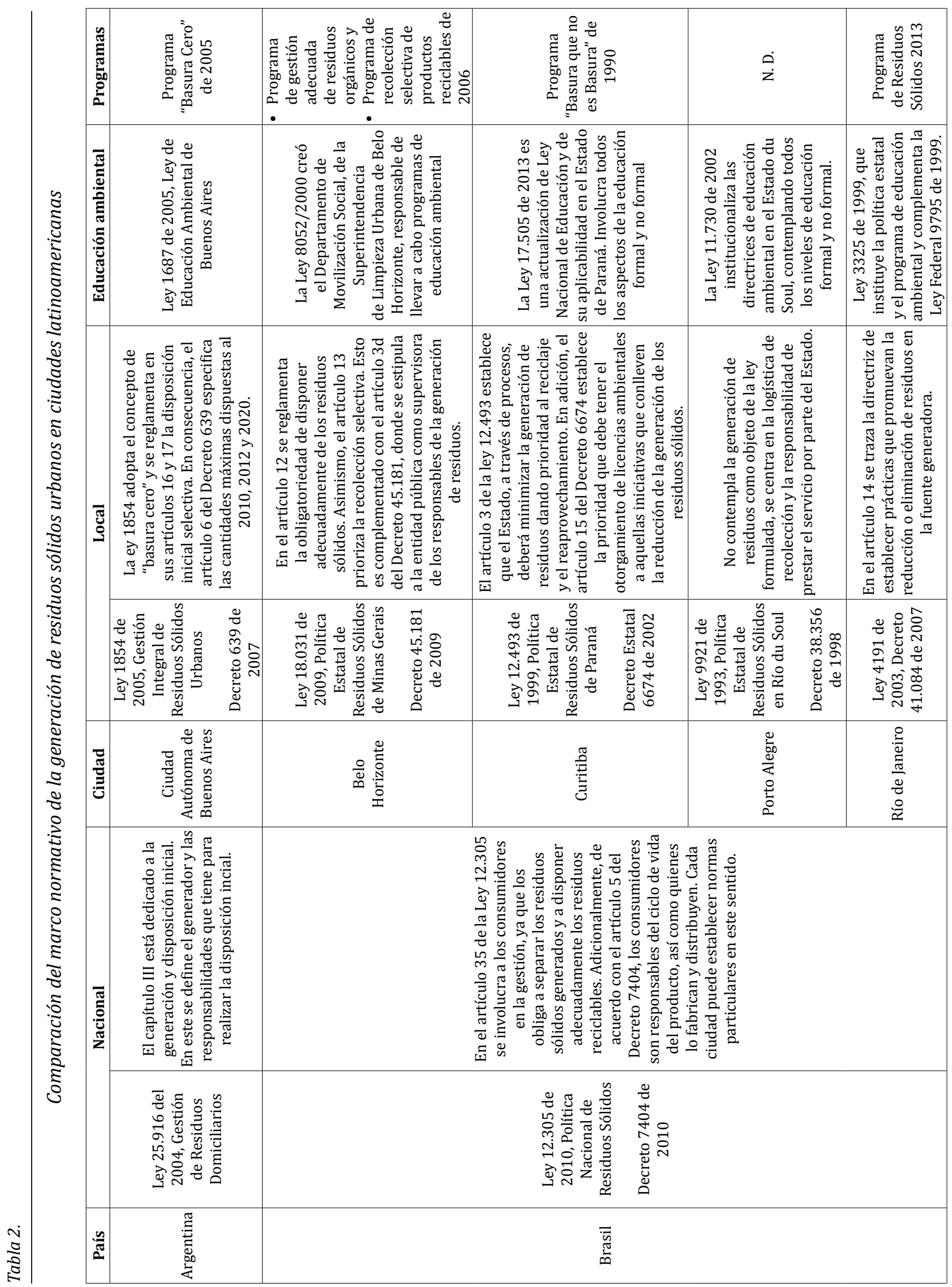




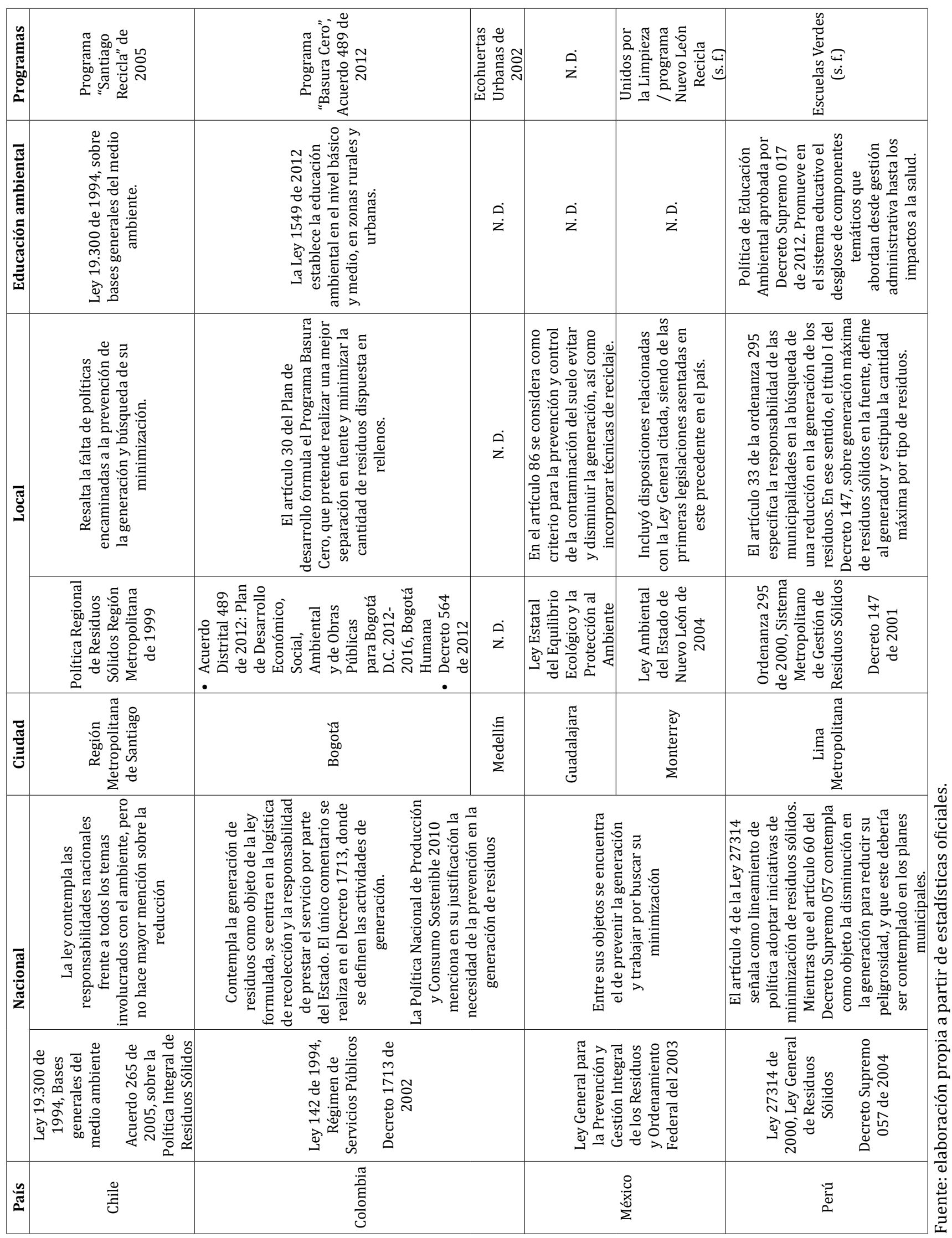


algunas variables como el PIB per cápita (como proxy de los ingresos promedio por habitante), las tasas de cobertura de educación primaria y secundaria (como proxy de educación ambiental), la actividad económica y el grado de urbanización son estadísticamente significativas para explicar el comportamiento de la generación de residuos sólidos urbanos.

En un primer ejercicio se pretendió replicar la metodología de los investigadores en mención; pero teniendo en cuenta la disponibilidad de las estadísticas, cuando se realizó el ejercicio del modelo de datos panel el conjunto de datos resultó no significativo para todas las variables seleccionadas. Además, en las ciudades de Guadalajara y Monterrey no fue posible hacer el análisis porque la información se encontraba a nivel estatal y no municipal.

La figura 1 muestra el comportamiento de la cantidad de residuos sólidos urbanos de cada ciudad en el periodo de análisis. Belo Horizonte es la única que presenta disminución progresiva a lo largo del tiempo, con un promedio de 0,43 ton/año por persona de residuos sólidos urbanos generados (cfr. tabla 3). Asimismo, la Ciudad Autónoma de Buenos Aires muestra que si bien del 2008 al 2011 aumentó la generación de los residuos, del 2012 hacia adelante ha presentado reducción, lo cual puede ser explicado por la aplicación del programa "Basura Cero".

\section{Figura 1.}

Residuos sólidos urbanos en ciudades seleccionadas, tonelada/año per cápita
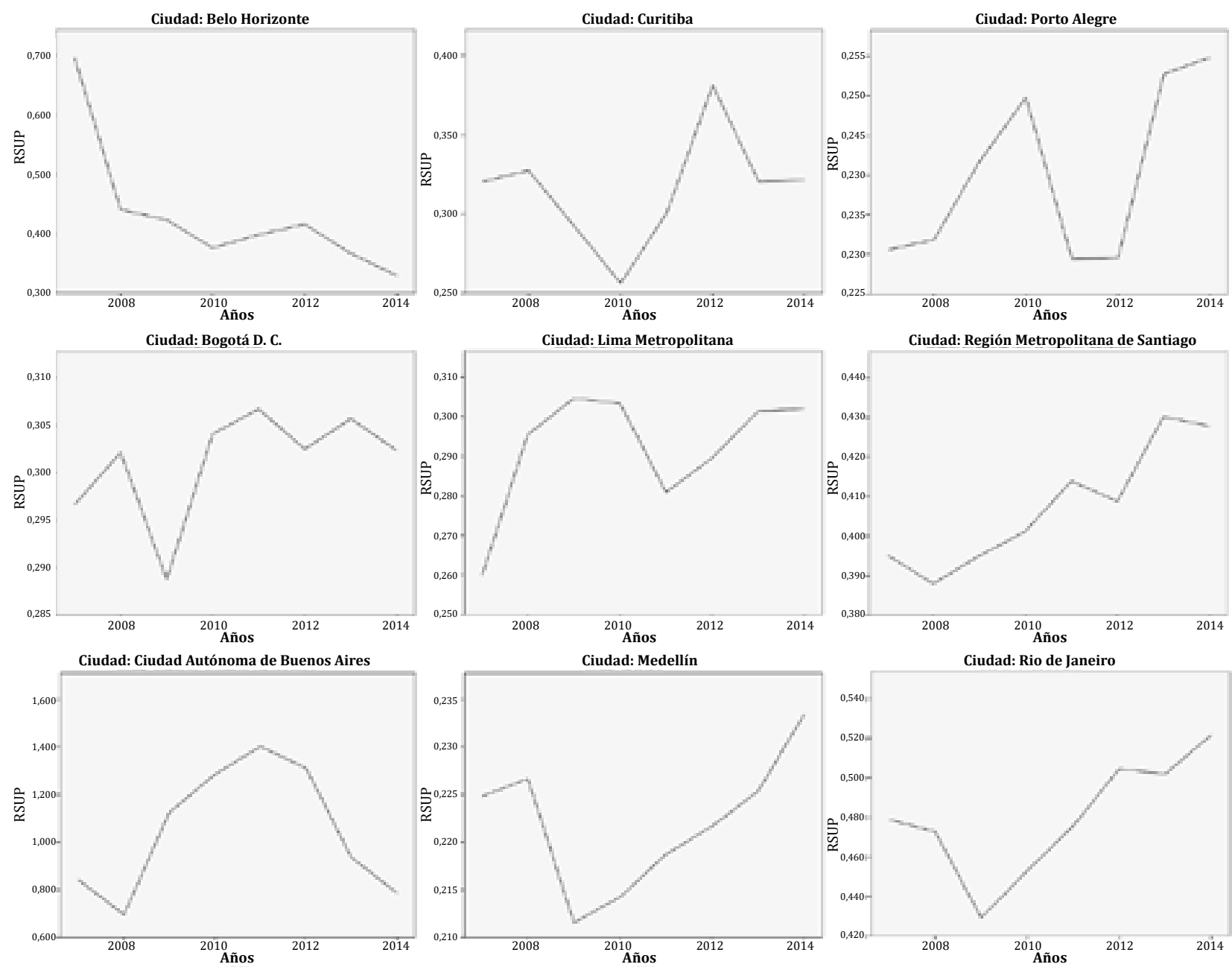

Fuente: elaboración propia a partir de estadísticas oficiales. 
Tabla 3.

Variables asociadas a los residuos sólidos urbanos generados en ciudades seleccionadas (tonelada/año per cápita)

\begin{tabular}{|c|c|c|c|c|c|c|c|}
\hline País & Ciudad & Variables & Rango & Mínimo & Máximo & Media & Desviación estándar \\
\hline \multirow{6}{*}{ Argentina } & \multirow{6}{*}{$\begin{array}{l}\text { Ciudad Autónoma de } \\
\text { Buenos Aires }\end{array}$} & RSUP & 0,71 & 0,70 & 1,40 & 1,05 & 0,27 \\
\hline & & PIBP & 5680,1 & $14.643,9$ & $20.324,0$ & $17.075,1$ & 2144,7 \\
\hline & & Población & 18.662 & 1.622 .915 & 1.641 .577 & 1.630 .116 & 7573 \\
\hline & & CG & 0,028 & 0,388 & 0,416 & 0,403 & 0,013 \\
\hline & & TCBP & 0,006 & 0,992 & 0,998 & 0,995 & 0,002 \\
\hline & & TCBS & 0,035 & 0,919 & 0,954 & 0,941 & 0,011 \\
\hline \multirow{24}{*}{ Brasil } & \multirow{6}{*}{ Belo Horizonte } & RSUP & 0,37 & 0,33 & 0,70 & 0,43 & 0,11 \\
\hline & & PIBP & $2.098,9$ & $13.930,3$ & $16.029,2$ & $15.129,4$ & 866,3 \\
\hline & & Población & 115.665 & 2.375 .444 & 2.491 .109 & 2.428 .417 & 43.211 \\
\hline & & CG & 0,08 & 0,49 & 0,56 & 0,51 & 0,02 \\
\hline & & TCBP & 0,19 & 1,07 & 1,26 & 1,17 & 0,06 \\
\hline & & TCBS & 0,14 & 0,88 & 1,02 & 0,96 & 0,05 \\
\hline & \multirow{6}{*}{ Curitiba } & RSUP & 0,12 & 0,26 & 0,38 & 0,32 & 0,04 \\
\hline & & PIBP & 5509,9 & $18.622,5$ & $24.132,4$ & $21.342,2$ & 2084,2 \\
\hline & & Población & 117.520 & 1.746 .896 & 1.864 .416 & 1.809 .784 & 44.426 \\
\hline & & CG & 0,089 & 0,453 & 0,542 & 0,493 & 0,030 \\
\hline & & TCBP & 0,164 & 1,059 & 1,223 & 1,120 & 0,060 \\
\hline & & TCBS & 0,123 & 0,861 & 0,984 & 0,927 & 0,050 \\
\hline & \multirow{6}{*}{ Porto Alegre } & RSUP & 0,03 & 0,23 & 0,25 & 0,24 & 0,01 \\
\hline & & PIBP & 2457,6 & $19.841,9$ & $22.299,5$ & $21.392,8$ & 869,5 \\
\hline & & Población & 62.543 & 1.409 .939 & 1.472 .482 & 1.433 .382 & 24.298 \\
\hline & & CG & 0,071 & 0,476 & 0,547 & 0,497 & 0,024 \\
\hline & & TCBP & 0,190 & 1,002 & 1,192 & 1,113 & 0,063 \\
\hline & & TCBS & 0,098 & 0,737 & 0,836 & 0,790 & 0,035 \\
\hline & \multirow{6}{*}{ Río de Janeiro } & RSUP & 0,09 & 0,43 & 0,52 & 0,48 & 0,03 \\
\hline & & PIBP & 2209,0 & $20.185,2$ & $22.394,2$ & $21.400,0$ & 714,0 \\
\hline & & Población & 360.210 & 6.093 .472 & 6.453 .682 & 6.299 .264 & 134.750 \\
\hline & & $\mathrm{CG}$ & 0,087 & 0,525 & 0,612 & 0,545 & 0,028 \\
\hline & & TCBP & 0,094 & 1,050 & 1,144 & 1,104 & 0,036 \\
\hline & & TCBS & 0,194 & 0,833 & 1,027 & 0,909 & 0,071 \\
\hline \multirow{6}{*}{ Chile } & \multirow{6}{*}{$\begin{array}{c}\text { Región Metropolitana de } \\
\text { Santiago }\end{array}$} & RSUP & 0,04 & 0,39 & 0,43 & 0,41 & 0,02 \\
\hline & & PIBP & 8636,2 & $16.620,7$ & $25.256,9$ & $21.329,0$ & 3071,5 \\
\hline & & Población & 587.884 & 6.640 .697 & 7.228 .581 & 6.931 .816 & 206.161 \\
\hline & & CG & 0,047 & 0,504 & 0,551 & 0,530 & 0,024 \\
\hline & & TCBP & 0,030 & 0,984 & 1,015 & 1,002 & 0,011 \\
\hline & & TCBS & 0,027 & 0,874 & 0,901 & 0,886 & 0,010 \\
\hline
\end{tabular}




\begin{tabular}{|c|c|c|c|c|c|c|c|}
\hline País & Ciudad & Variables & Rango & Mínimo & Máximo & Media & Desviación estándar \\
\hline \multirow{12}{*}{ Colombia } & \multirow{6}{*}{ Bogotá } & RSUP* & 0,02 & 0,29 & 0,31 & 0,30 & 0,01 \\
\hline & & PIBP** & 4419,7 & $16.066,4$ & $20.486,1$ & $17.802,1$ & 1542,8 \\
\hline & & Población & 726.617 & 7.050 .228 & 7.776 .845 & 7.414 .877 & 254.345 \\
\hline & & $\mathrm{CG}^{* * *}$ & 0,009 & 0,522 & 0,531 & 0,526 & 0,004 \\
\hline & & ТСВР• & 0,135 & 0,953 & 1,088 & 1,031 & 0,056 \\
\hline & & TCBS•• & 0,046 & 1,045 & 1,091 & 1,069 & 0,015 \\
\hline & \multirow{6}{*}{ Medellín } & RSUP & 0,02 & 0,21 & 0,23 & 0,22 & 0,01 \\
\hline & & PIBP & 5760,9 & $13.304,9$ & $19.065,8$ & $16.009,4$ & 2113,1 \\
\hline & & Población & 175.879 & 2.265 .244 & 2.441 .123 & 2.354 .594 & 61.629 \\
\hline & & $\mathrm{CG}$ & 0,043 & 0,500 & 0,543 & 0,522 & 0,016 \\
\hline & & TCBP & 0,269 & 1,000 & 1,269 & 1,203 & 0,088 \\
\hline & & TCBS & 0,155 & 1,000 & 1,155 & 1,109 & 0,051 \\
\hline \multirow{6}{*}{ Perú } & \multirow{6}{*}{ Lima Metropolitana } & RSUP & 0,04 & 0,26 & 0,30 & 0,29 & 0,02 \\
\hline & & PIBP & 3283,9 & 9586,3 & $12.870,2$ & $11.203,9$ & 1073,5 \\
\hline & & Población & 992.828 & 8.758 .889 & 9.751 .717 & 9.242 .223 & 347.988 \\
\hline & & CG & 0,060 & 0,400 & 0,460 & 0,425 & 0,019 \\
\hline & & TCBP & 0,045 & 0,900 & 0,945 & 0,925 & 0,014 \\
\hline & & TCBS & 0,034 & 0,837 & 0,871 & 0,858 & 0,012 \\
\hline
\end{tabular}

*RSUP: Residuos Sólidos Urbanos per cápita, **PIBP: PIB per-cápita a PPA, ${ }^{* * *}$ CG: Coeficiente de Gini, •TCBP: Tasa de Cobertura Bruta Primaria, $\bullet$ TCBS: Tasa de Cobertura Bruta Secundaria Fuente: elaboración propia a partir de estadísticas oficiales.

Se destaca igualmente el caso de Curitiba, ciudad que, además de presentar avances en la normatividad, desarrolla acciones concretas para mejorar la gestión de los residuos. Este hecho se evidencia en una generación promedio de 0,32 ton/año por habitante, y es más notorio cuando se compara con la Ciudad Autónoma de Buenos Aires, que con un tamaño poblacional similar (1.809.784 y 1.630 .116 habitantes en promedio, respectivamente) genera más del doble que Curitiba (tabla 3).

Por otro lado, Lima Metropolitana y Bogotá D.C. (las ciudades más grandes, con 9.242 .223 y 7.414.877 de habitantes en promedio, respectivamente) producen tres veces menos toneladas de residuos per cápita en comparación con los de la Ciudad Autónoma de Buenos Aires. Estas dos ciudades, en casi todo el periodo de estudio, muestran que la generación per cápita se mantuvo constante en 0,29 y 0,30 ton/año per cápita, respectivamente. A pesar de que no es un resultado satisfactorio, sí es mejor que el de Río de Janeiro, Medellín y la Región
Metropolitana de Santiago, donde la generación ha aumentado (figura 2).1

La Región Metropolitana de Santiago pasó de generar 0,40 a 0,43 ton/año per cápita entre el 2007 y el 2014. Una posible explicación de este comportamiento se encuentra en el PIB per cápita a PPA, que presentó una tasa de crecimiento promedio de $5,36 \%$. En este sentido, es posible argumentar que mayores niveles de ingreso se traducen en un mayor consumo, reflejado a su vez en una mayor cantidad de residuos sólidos generados. En la figura 2 se observa la relación directa entre ambas variables.

En contraste con la teoría, en Belo Horizonte el PIB per cápita a PPA crece durante el periodo de estudio, mientras que la generación de residuos sólidos urbanos disminuye (figura 3). Se destaca en esta ciudad la organización e inclusión de los recicladores de oficio y la creación de conciencia sobre la separación en la fuente, lo que se ha traducido en reducciones en la generación de 0,37 ton/año, al pasar de 0,70 en el 2007 a 0,33 ton/año por habitante en el 2014. 
Figura 2.

Residuos sólidos urbanos y PIB per cápita a PPA en Región Metropolitana de Santiago

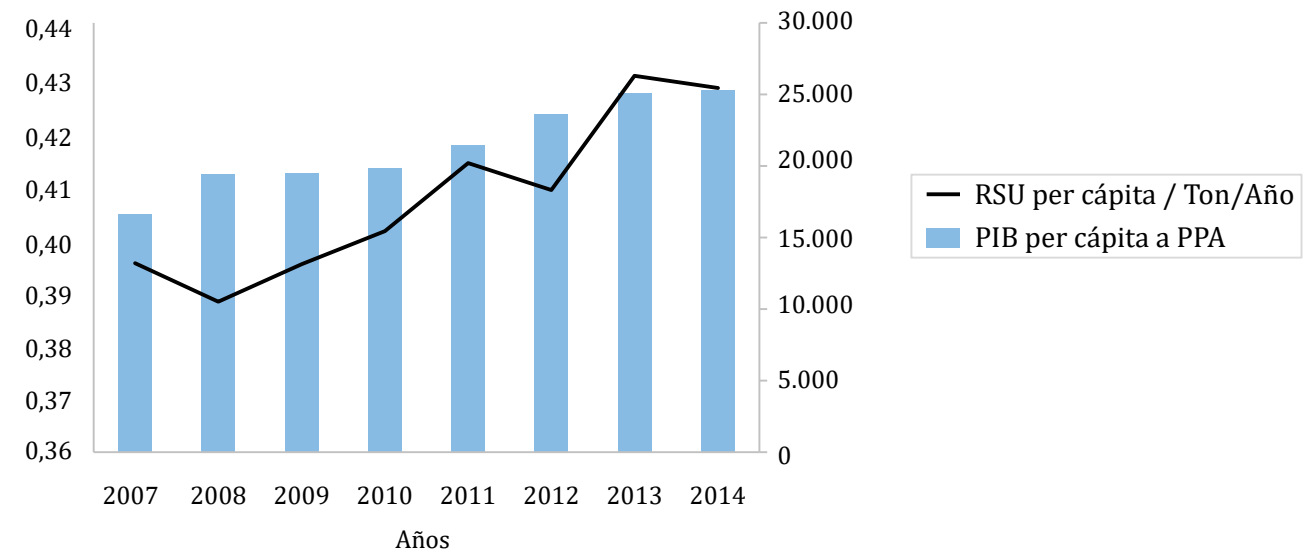

Fuente: elaboración propia a partir de estadísticas oficiales.

Figura 3.

Residuos sólidos urbanos y PIB per cápita a PPA en Belo Horizonte

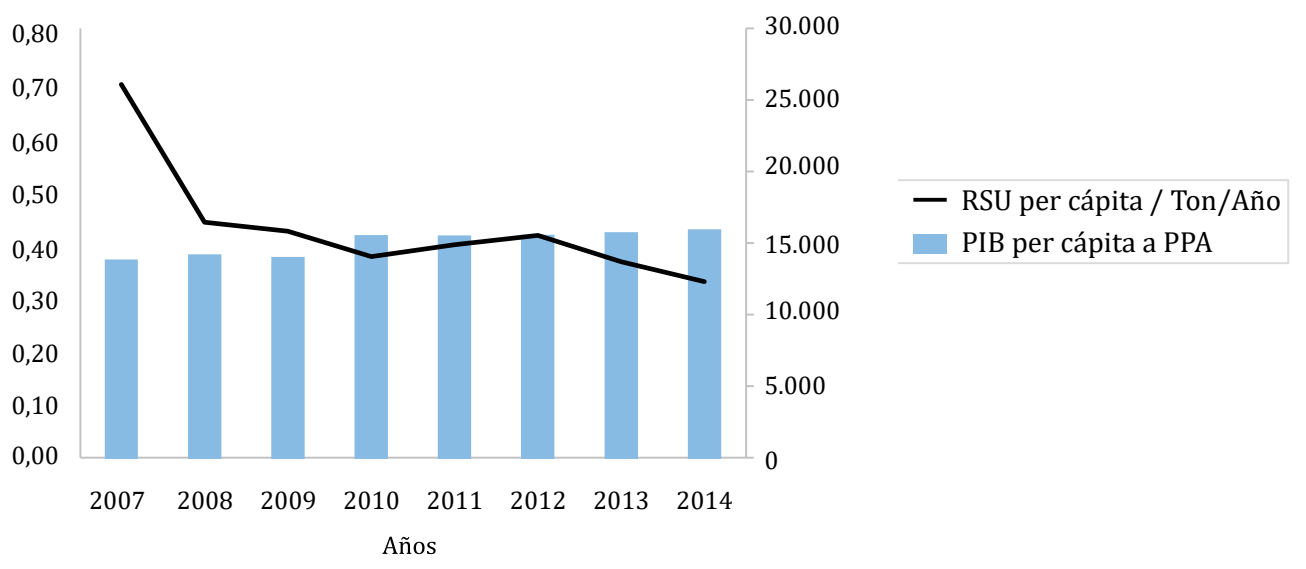

Fuente: elaboración propia a partir de estadísticas oficiales.

Por su parte, el PIB per cápita a PPA creció en promedio durante el periodo un $1,77 \%$, pasando de 13.930 a 16.029 . Este mismo resultado se corrobora en el informe realizado por la Economist Intelligence Unit (2010): “Un hallazgo sorprendente al examinar los resultados generales es que no existe relación clara entre desempeño ambiental y nivel de ingreso de la ciudad, el cual fue determinado como PIB promedio por persona en este estudio" (p. 11).

Ahora bien, en el análisis de las variables asociadas a la generación de residuos sólidos urbanos, la figura 4 muestra que no es posible concluir una correlación en las ciudades estudiadas, es decir, no hay tendencia entre las ciudades a la hora de analizar la relación positiva o negativa de estas variables con la generación de residuos sólidos urbanos.

Como se mencionó, se aplicó la prueba de normalidad Kolmogorov-Smirnov, y se determinó que el coeficiente de Pearson es la mejor medida del grado de asociación lineal existente entre las variables. Los resultados contenidos en la tabla 4 respaldan lo visualizado en la figura 4, en la medida 
Correlaciones por ciudad

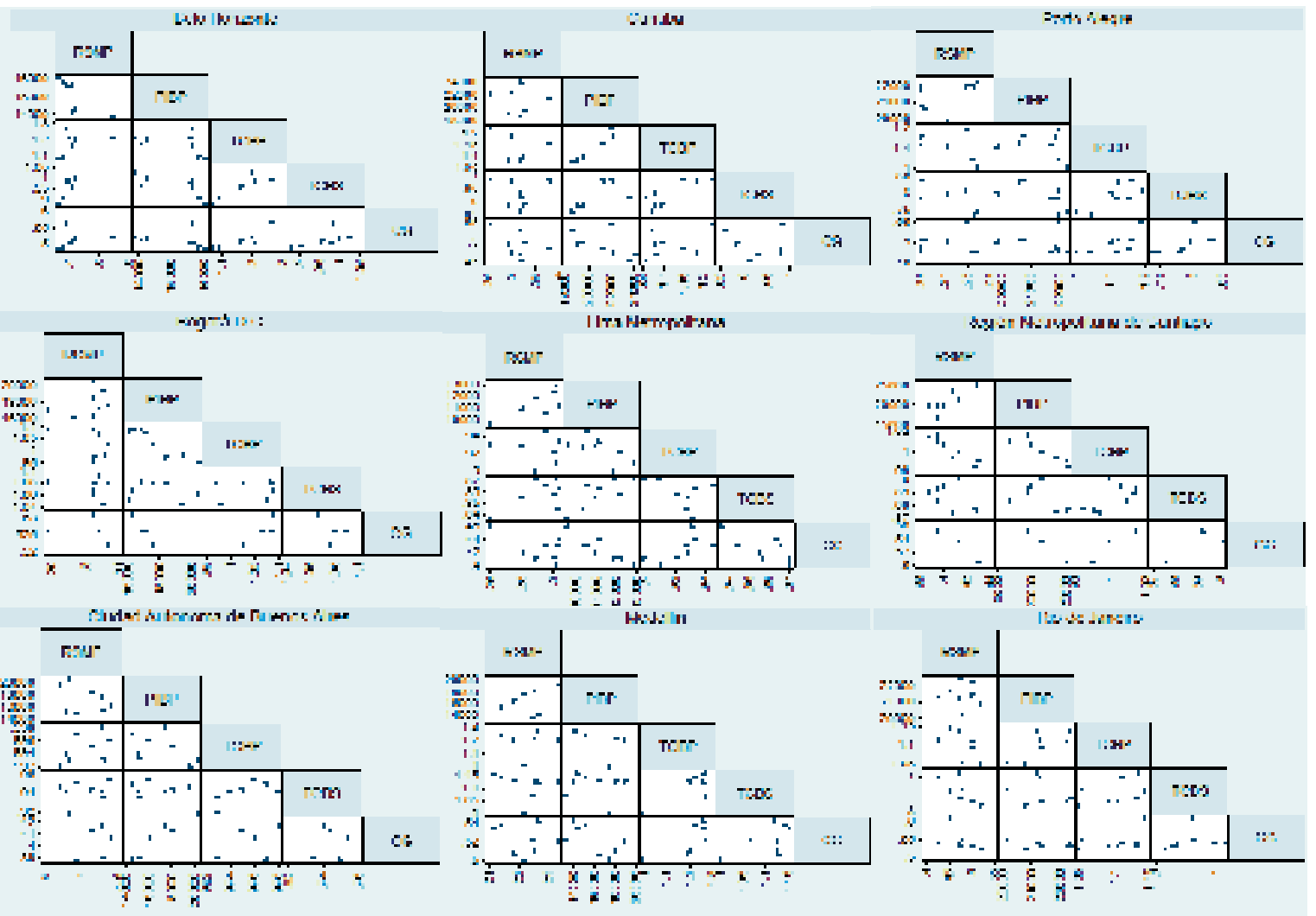

Fuente: elaboración propia a partir de estadísticas oficiales.

en que ninguna variable tiene un comportamiento homogéneo en todas las ciudades, además de que en muy pocas existe nivel de significancia.

Los resultados corroboran lo dicho con anterioridad sobre la correlación positiva fuerte $(0,886)$, con un nivel de significancia del 0,01 a dos colas, entre la generación de residuos sólidos urbanos por habitante y el nivel de ingresos medido a través del PIB per cápita a PPA para la Región Metropolitana de Santiago; sin embargo, para las demás ciudades no se puede afirmar lo mismo ya que no hay significancia estadística.

Por otro lado, la cobertura en educación tanto básica como secundaria forma parte de las variables que se relacionan con la generación. Se asume teóricamente una relación negativa. Solo Río de Janeiro tiene un nivel de significancia del 0,05 a dos colas y la Región Metropolitana de Santiago tiene un nivel de significancia de 0,01 , lo cual es signo de una relación negativa moderada para la educación media y negativa fuerte en básica, respectivamente. Por su parte, Belo Horizonte muestra una relación positiva moderada, lo cual indica nuevamente que no es posible generalizar la variable.

Un limitante de los datos sobre cobertura en educación radica en la imposibilidad de verificar si estos procesos de enseñanza se vinculan de manera transversal o directa con la educación ambiental $y$, en especial, con el cambio de conciencia sobre la producción limpia, el consumo responsable o la forma de disposición de los residuos sólidos.

Ahora bien, a partir de la exploración realizada en las ciudades, se encontró un común denominador: 
Coeficiente de Pearson

\begin{tabular}{|c|c|c|c|c|c|}
\hline Ciudad & Estadísticos & PIBP & TCBP & TCBS & CG \\
\hline \multirow{2}{*}{ Belo Horizonte } & Correlación de Pearson & $-0,737^{*}$ & 0,343 & $0,715^{*}$ & 0,174 \\
\hline & Sig. (bilateral) & 0,037 & 0,405 & 0,046 & 0,680 \\
\hline \multirow{2}{*}{ Bogotá } & Correlación de Pearson & 0,423 & $-0,506$ & $-0,091$ & $-0,126$ \\
\hline & Sig. (bilateral) & 0,296 & 0,201 & 0,831 & 0,767 \\
\hline \multirow{2}{*}{ Ciudad Autónoma de Buenos Aires } & Correlación de Pearson & $-0,360$ & 0,513 & 0,248 & $0,851^{* *}$ \\
\hline & Sig. (bilateral) & 0,381 & 0,194 & 0,553 & 0,007 \\
\hline \multirow{2}{*}{ Curitiba } & Correlación de Pearson & $-0,016$ & $-0,348$ & $-0,043$ & $-0,455$ \\
\hline & Sig. (bilateral) & 0,969 & 0,399 & 0,919 & 0,258 \\
\hline \multirow{2}{*}{ Lima Metropolitana } & Correlación de Pearson & 0,585 & $-0,093$ & 0,566 & $-0,560$ \\
\hline & Sig. (bilateral) & 0,128 & 0,827 & 0,144 & 0,149 \\
\hline \multirow{2}{*}{ Medellín } & Correlación de Pearson & 0,438 & $-0,084$ & $-0,236$ & $-0,218$ \\
\hline & Sig. (bilateral) & 0,277 & 0,843 & 0,573 & 0,604 \\
\hline \multirow{2}{*}{ Porto Alegre } & Correlación de Pearson & 0,399 & $-0,582$ & $-0,645$ & 0,067 \\
\hline & Sig. (bilateral) & 0,327 & 0,130 & 0,084 & 0,875 \\
\hline \multirow{2}{*}{ Región Metropolitana de Santiago } & Correlación de Pearson & $0,886^{* *}$ & $-0,941^{* *}$ & $-0,456$ & 0,264 \\
\hline & Sig. (bilateral) & ,003 & 0,000 & 0,257 & 0,528 \\
\hline \multirow{2}{*}{ Río de Janeiro } & Correlación de Pearson &,- 055 & $-0,564$ & $-0,743^{*}$ & $-0,525$ \\
\hline & Sig. (bilateral) & ,897 & 0,145 & 0,035 & 0,181 \\
\hline
\end{tabular}

* La correlación es significativa en el nivel 0,05 ( 2 colas). ** La correlación es significativa en el nivel 0,01 (2 colas). Fuente: elaboración propia a partir de estadísticas oficiales.

Las personas no participan activamente en la correcta gestión de los residuos porque no conocen las consecuencias a la salud y a su bienestar por un mal manejo no solo en el hogar sino en su entorno, porque desconocen cómo manejarlos o porque no les importa y no quieren tomarse la molestia de hacer un esfuerzo en manejarlos correctamente como su separación, solo presentan en la calle sus bolsas o recipientes y esperan que el camión recolector pase por ellos sin ninguna responsabilidad. (Gobierno del Estado de Nueva León, 2011, p. 31)

Según la síntesis presentada en la tabla 5 , se enfatiza en la necesidad que tiene la región de trabajar por traducir la política de gestión de residuos sólidos en acciones que transformen la realidad y se conviertan en verdaderas alternativas sostenibles; para ello, lo local se podría seguir potencializando como escenario de transformación. Ciertamente, hay que aprender de experiencias como la de Curitiba y enfatizar en la necesidad de expandir la educación ambiental a toda la población. La Red de Desarrollo Sostenible (2018) lo expresa así:

Los mejores servicios de gestión de residuos sólidos urbanos en América Latina dependerán de que las administraciones municipales fortalezcan su capacidad de respuesta al manejo de los residuos. [...] Su función debe ir más allá de esto, a la gestión integrada del material de desecho a lo largo de su ciclo de vida. Los municipios también deben involucrarse en discusiones más amplias, tales como una mejor administración de los productos entre los fabricantes y los minoristas. 
Tabla 5.

Síntesis de resultados de la comparación entre ciudades de la gestión de residuos sólidos domiciliarios

\begin{tabular}{|c|c|c|c|}
\hline Ciudad & $\begin{array}{c}\text { Programas de } \\
\text { aprovechamiento }\end{array}$ & Topes de generación & $\begin{array}{c}\text { Normativa y programas de } \\
\text { educación ambiental }\end{array}$ \\
\hline Ciudad Autónoma de Buenos Aires & Sí & Sí & Alto \\
\hline Belo Horizonte & Sí & N. d. & Medio \\
\hline Curitiba & Sí & Sí & Medio \\
\hline Porto Alegre & N. $d^{*}$ & N. d & Medio \\
\hline Río de Janeiro & N.d. & N.d. & Medio \\
\hline Región Metropolitana de Santiago & Sí & N. d. & Medio \\
\hline Bogotá D.C. & Sí & N. d. & Medio \\
\hline Medellín & Sí & N. d. & Bajo \\
\hline Lima Metropolitana & Sí & Sí & Medio \\
\hline
\end{tabular}

*N. d.: no disponible.

Fuente: elaboración propia.

Finalmente, aunque la lógica en las ciudades se encamina a intentar recircular los residuos para que vuelvan a forman parte del ciclo productivo, incorporando programas que reduzcan la generación - como es el caso de "Basura Cero"-, existen indicios para afirmar que la logística del aprovechamiento no parece ser lo suficientemente robusta para introducir de nuevo los residuos como materia prima del proceso productivo. Además, las acciones se dirigen con más ahínco a resolver los problemas de disposición y menos a los cambios comportamentales de los individuos.

\section{CONCLUSIONES}

El estudio de la generación de residuos sólidos urbanos busca aportar a la consecución del desarrollo sostenible. Para ello, se requiere de políticas claras, una normativa que incentive el adecuado manejo y unos ciudadanos conscientes de la necesidad de cambiar sus hábitos tanto en los procesos de producción como de consumo. Sin embargo, la falta de información histórica confiable impide hacer un adecuado seguimiento a los avances que en esta materia se hayan podido lograr.

Por lo anterior, es recomendable que dentro de los planes de gestión de manejo de residuos sólidos se establezcan con claridad las variables y los indicadores que se deben construir, desde lo cuantitativo y de lo cualitativo, para la toma de decisiones y el ajuste de la política. Si esto se logra, es posible de hacer análisis inferencial que permita determinar las causas de la generación y así la política pública se podría enfocar en solucionar las problemáticas particulares.

Es imperante que Bogotá aprenda de los casos en los cuales la normativa no se quedó solo en el deber ser, sino que a través de las acciones colectivas permitieron evidenciar cambios que son exaltables. En general, las ciudades analizadas presentan avances, pero aún son muchos los retos que deben afrontar, y uno de ellos es el relacionado con la aplicación de programas efectivos para concientizar a los ciudadanos de la necesidad de generar una menor cantidad de residuos sólidos. 


\section{REFERENCIAS}

1. Banco Interamericano de Desarrollo (BID), Asociación Interamericana de Ingeniería Sanitaria y Ambiental (AIDIS) y Organización Panamerica de la Salud (OPS) (2011). Informe de la evaluación regional del manejo de residuos sólidos urbanos en América Latina y el Caribe 2010. Washington: Autor.

2. Boisier, S. (1999). Desarrollo (local): ¿de qué estamos hablando? https://flacsoandes.edu.ec/web/imagesF TP/1245948918.Desarrollo_Local_De_que_estamos_hablando_2_.pdf

3. Comisión Mundial sobre Medio Ambiente y el Desarrollo (1987). Informe Brundtland: Nuestro Futuro Común. Oxford: Oxford University Press.

4. Dueñas, J. C. (2011). El papel de la educación ante el desarrollo sostenible. Transatlántica de Educación, 6(9), 16-19.

5. Economist Intelligence Unit (2010). Índice de ciudades verdes de América Latina. Múnich: Siemens AG.

6. Gobierno de Chile, Comisión Nacional de Medio Ambiente (CONAMA) (2010). Primer reporte del manejo de residuos sólidos en Chile. Santiago de Chile: Autor. http://www.sinia.cl/1292/articles-49564_in forme_final.pdf

7. Gobierno del Estado de Nueva León (2011). Programa Estatal de Gestión Integral de Residuos de Nuevo León 2009-2015. Monterrey: Autor.

8. Greenpeace (2014, 24 de julio). Greenpeace: los logros de la Ley de Basura Cero empiezan a aparecer. http://www.greenpeace.org/argentina/es/noticias/Greenpeace-los-logros-de-la -Ley-de-Basura -Cero-empiezan-a-aparecer/

9. Mullahy, L. (1993). Experiencias brasileñas a nivel local: tres ideas para deshacerse del problema de la basura. Ambiente y Desarrollo, 1993, 61-66.

10. Municipalidad Metropolitana de Lima (2014, 22 de julio). Plan Integral de Gestión Ambiental de Residuos Sólidos de la Provincia de Lima 2015-2025. Ordenanza 1803. https:/pruebafuerzasocial.files.wordpress. com/2015/05/plan-integral-de-gestic3b3n-ambiental-de-residuos-sc3b3lidos-de-la-provincia-de-lima.pdf

11. Red de Desarrollo Sostenible (2018, 14 de enero). Los residuos sólidos urbanos. https://rds.org.co/es/ novedades/los-residuos-solidos-urbanos

12. Sen, A. (2001). Desarrollo y libertad. Bogotá: Planeta.

13. Tello, M. D. (2006) Las teorías del desarrollo local y la teoría y práctica del proceso de desentralización en los países en desarrollo. Lima: Pontificia Universidad Catolica del Perú.

14. Usaquén, M. y Sánchez, M. (2014). Determinantes de la generación de residuos sólidos en diez municipios representativos del departamento de Cundinamarca, 2007-2012. Revista Criterio Libre, 12(20), 140-161. https://doi.org/10.18041/1900-0642/criteriolibre.2014v12n20.184 